\title{
Atorvastatin attenuates TGF- $\beta 1$-induced fibrogenesis by inhibiting Smad3 and MAPK signaling in human ventricular fibroblasts
}

\author{
YANFEI DU ${ }^{1 *}$, HAIYING XIAO ${ }^{2 *}$, JUN WAN $^{3}$, XINYU WANG $^{2}$, TAO LI $^{2}$, SHUZHAN ZHENG $^{1}$, \\ JIAN FENG ${ }^{1}$, QIANG YE ${ }^{1}$, JIAFU LI ${ }^{1}$, GUANG LI ${ }^{2}$ and ZHONGCAI FAN ${ }^{1}$ \\ ${ }^{1}$ Key Laboratory of Medical Electrophysiology, Ministry of Education, \\ Department of Cardiology, The Affiliated Hospital of Southwest Medical University; \\ ${ }^{2}$ Key Laboratory of Medical Electrophysiology, Ministry of Education, Institute of Cardiovascular Research, \\ Southwest Medical University; ${ }^{3}$ Department of Basic Medical Sciences, College of Basic Medical Sciences, \\ Southwest Medical University, Luzhou, Sichuan 646000, P.R. China
}

Received November 20, 2019; Accepted May 6, 2020

DOI: $10.3892 / \mathrm{ijmm} .2020 .4607$

\begin{abstract}
Excessive proliferation and myofibroblasts transformation of cardiac fibroblasts play a critical role in the process of cardiac fibrosis. Atorvastatin (ATV), a 3-hydroxy-3-methyl-glutaryl-coenzyme A reductase inhibitor, is commonly used to treat hypercholesterolemia. It has previously been shown that ATV has potential anti-fibrotic effects. However, the underlying mechanisms of ATV against cardiac fibrosis remain to be fully elucidated, and to the best of our knowledge, there are no reports focusing on the effects of ATV on transforming growth factor- $\beta 1$ (TGF- $\beta 1$ )-induced human ventricular fibroblasts (hVFs) activation. In the present study, hVFs were stimulated with TGF- $\beta 1$ with or without pretreatment with ATV. Subsequently, hVF proliferation, cytotoxicity, myofibroblast differentiation and pro-fibrotic gene expression were assessed. Canonical and non-canonical signaling downstream of TGF- $\beta 1$, such as Smad3 and mitogen-activated protein kinase (MAPK) signaling, were investigated by evaluating the phosphorylation levels of Smad3, extracellular
\end{abstract}

Correspondence to: Professor Zhongcai Fan, Key Laboratory of Medical Electrophysiology, Ministry of Education, Department of Cardiology, The Affiliated Hospital of Southwest Medical University, 25 Taiping Street, Luzhou, Sichuan 646000, P.R. China E-mail: fanzhongcaixnyd@126.com

Professor Guang Li, Key Laboratory of Medical Electrophysiology, Ministry of Education, Institute of Cardiovascular Research, Southwest Medical University, 319 Zhongshan Road, Luzhou, Sichuan 646000, P.R. China

E-mail: liguang7645@126.com

*Contributed equally

Key words: cardiac fibrosis, human ventricular fibroblasts, transforming growth factor- $\beta 1$, atorvastatin, extracellular matrix, signaling pathways signal-regulated kinase 1/2, p38 MAPK and c-Jun N-terminal kinase. The results indicated that ATV significantly prevented TGF- $\beta 1$-induced cell proliferation, myofibroblast differentiation and production of extracellular matrix proteins, such as matrix metalloproteinase-2, collagen I and collagen III, in hVFs. Furthermore, ATV effectively inhibited TGF- $\beta 1$-induced activation of Smad3 and MAPK signaling in hVFs. In conclusion, the present results demonstrated that ATV prevented TGF- $\beta 1$-induced fibrogenesis in hVFs, at least in part by inhibiting the Smad3 and MAPK signaling pathways. Therefore, these results imply that ATV may be a promising agent to treat myocardial fibrosis.

\section{Introduction}

Myocardial fibrosis is a common pathological feature of multiple end-stage cardiovascular diseases, including hypertension, advanced coronary heart disease and cardiomyopathy (1). Fibrosis is defined by overproliferation and activation of cardiac fibroblasts (CFs), and accumulation of extracellular matrix (ECM) components secreted by activated fibroblasts $(2,3)$. Therefore, prevention of CF proliferation and abrogation of $\mathrm{CF}$ trans-differentiation into myofibroblasts may become an effective strategy for treating cardiac fibrosis.

Transforming growth factor- $\beta 1$ (TGF- $\beta 1$ ) is a major pro-fibrotic factor $(2,4)$. It can stimulate the proliferation of $\mathrm{CFs}$ and the differentiation of CFs into myofibroblasts, which are characterized by the upregulation of $\alpha$-smooth muscle actin $(\alpha-S M A)$ and the secretion of ECM proteins, such as collagen I and collagen III $(5,6)$. TGF- $\beta 1$ ligand initiates a signaling cascade through cell-surface receptors and intracellular Smad signal proteins, such as the canonical signal transducer-Smad2/3 protein, whose activation is associated with the transcription of numerous pro-fibrotic genes $(7,8)$. Furthermore, TGF- $\beta 1$ can induce other non-canonical signaling cascades independently of Smads, such as mitogen-activated protein kinase (MAPK) signaling pathways, including extracellular signal-regulated kinase 1/2 (ERK1/2), p38 MAPK and c-Jun N-terminal kinase (JNK) (9). Accumulating evidence has demonstrated that 
MAPK signaling plays a critical role in ECM synthesis and in the proliferation of CFs $(10,11)$. Thus, pharmacological interventions in these signaling pathways could be considered as a promising therapeutic strategy against cardiac fibrosis.

Statins, which are inhibitors of the 3-hydroxy-3-methylglutaryl-coenzyme A reductase, have been extensively applied to treat patients with hypercholesterolemia in the past decade due to their lipid-reducing effects (12). Furthermore, statins have also been reported to possess pleiotropic cardiovascular effects, including reduced oxidative stress and inflammation, decreased platelet adhesion, improved endothelial function and enhanced atherosclerotic plaque stability (13-15). In addition, previous studies have shown that atorvastatin (ATV), a member of the statin family, exerts potential anti-myocardial fibrotic properties (16-20). Nevertheless, to the best of our knowledge, the molecular mechanism involved in the effect of ATV on cardiac fibrosis remains to be clarified, and limited information is available concerning the possible impact of ATV on TGF- $\beta 1$-induced human ventricular fibroblast (hVF) proliferation and myofibroblast differentiation. Thus, the aim of the present study was to investigate the impact of ATV on TGF- $\beta 1$-stimulated fibrogenesis and its underlying molecular mechanism in hVFs. The findings indicated that ATV prevents TGF- $\beta 1$-induced fibrogenesis in hVFs, at least in part, by inhibiting the Smad3 and MAPK signaling pathways.

\section{Materials and methods}

Cells, chemicals and reagents. Adult hVFs (cat. no. 6310) and complete medium for their culture (fibroblast medium-2; FM-2; cat. no. 2331) were obtained from ScienCell Research Laboratories, Inc. Antibodies against $\alpha$-SMA, matrix metalloproteinase-2 (MMP-2), collagen I and collagen III were purchased from ProteinTech Group, Inc. Antibodies against phosphorylated (p)-Smad3, total (t)-Smad3, p-ERK1/2, t-ERK1/2, p-JNK, t-JNK, p-p38 and t-p38 were purchased from Cell Signaling Technology, Inc. Anti-GAPDH antibody and Cell Counting Kit-8 (CCK-8) were purchased from Beyotime Institute of Biotechnology. ATV was obtained from Sigma-Aldrich (Merck KGaA). Recombinant human TGF- $\beta 1$ cytokine was purchased from PeproTech, Inc.

Cell culture. hVFs were maintained in FM-2 supplemented with 5\% fetal bovine serum (cat. no. 0025; ScienCell Research Laboratories, Inc.) and $1 \%$ penicillin-streptomycin in a humidified atmosphere with $5 \% \mathrm{CO}_{2}$ at $37^{\circ} \mathrm{C}$. The cells were passaged $\leq 8$ times and were cultured for $16 \mathrm{~h}$ in serum-free medium before treatment. In the present study, an in vitro cardiac fibrosis model was established by treatment of hVFs with TGF- $\beta 1(5 \mathrm{ng} / \mathrm{ml})$ for $24 \mathrm{~h}$ at $37^{\circ} \mathrm{C}$ in a humidified atmosphere with $5 \% \mathrm{CO}_{2}$, according to the previous literature $(3,21)$.

Cell viability assays. The survival rate of the cells was determined by CCK-8 assay according to the manufacturer's protocol. In brief, fibroblasts $\left(1 \times 10^{4}\right.$ cells/well) were seeded into 96-well plates until $\sim 90 \%$ confluence. Subsequently, cells were subjected to serum starvation for $16 \mathrm{~h}$, followed by treatment with ATV $(0,2,5,10,20$ and $30 \mu \mathrm{M})$ for $24 \mathrm{~h}$ at $37^{\circ} \mathrm{C}$. Following treatment, the cells were washed with PBS, and then $10 \mu \mathrm{lCCK}-8$ was added to each well and incubated for an additional $4 \mathrm{~h}$ at $37^{\circ} \mathrm{C}$.
Next, the absorbance of each well at $450 \mathrm{~nm}$ was examined using an automatic microplate reader (TECAN M200; Tecan Group, Ltd.). The results were expressed as percentages of the control group (cells were treated with an equal amount of DMSO).

Cell proliferation assay. hVFs were seeded into 96-well plates at $2 \times 10^{3}$ cells/well and cultured overnight. Cells were deprived of serum for $16 \mathrm{~h}$ before being pretreated with ATV $(2,5$ and $10 \mu \mathrm{M}$ ) or DMSO for $3 \mathrm{~h}$, followed by stimulation with TGF- $\beta 1(5 \mathrm{ng} / \mathrm{ml})$ for $24 \mathrm{~h}$ in a humidified atmosphere with $5 \% \mathrm{CO}_{2}$ at $37^{\circ} \mathrm{C}$. Subsequently, the medium was removed from each well, and CCK-8 solution ( $10 \mu \mathrm{l} /$ well) was added into the wells, followed by incubation for an additional $4 \mathrm{~h}$ at $37^{\circ} \mathrm{C}$. Finally, the optical density of each well was detected at an absorbance wavelength of $450 \mathrm{~nm}$ using the aforementioned microplate reader.

Western blotting. hVFs $\left(5 \times 10^{5}\right.$ cells/well $)$ were plated into 6 -well plates, cultured to $\sim 80 \%$ confluence and starved of serum for $16 \mathrm{~h}$. Then, the cells were pretreated for $3 \mathrm{~h}$ with ATV $(10 \mu \mathrm{M})$ or DMSO before treatment with $5 \mathrm{ng} / \mathrm{ml}$ TGF- $\beta 1$ for $24 \mathrm{~h}$ at $37^{\circ} \mathrm{C}$. Subsequently, the cells were washed twice with cold PBS and lysed in RIPA lysis buffer (Beyotime Institute of Biotechnology) at $4^{\circ} \mathrm{C}$. Lysates were centrifuged at $13,000 \mathrm{x} \mathrm{g}$ for $15 \mathrm{~min}$ at $4^{\circ} \mathrm{C}$, and protein concentrations were determined by BCA assay (cat. no. 23227; Pierce; Thermo Fisher Scientific, Inc.). Cell lysates $(30 \mu \mathrm{g})$ were separated by $8-10 \%$ SDS-PAGE and transferred onto a polyvinylidene difluoride membrane (Merck KGaA). The membranes were blocked with blocking buffer (5\% nonfat dry milk in TBS plus $0.1 \%$ Tween-20) for $1 \mathrm{~h}$ at room temperature and incubated with primary antibodies overnight at $4^{\circ} \mathrm{C}$, including antibodies against $\alpha$-SMA (1:1,000; cat. no. 14395-1-AP), MMP-2 (1:1,000; cat. no. 10373-2-AP), collagen I (1:2,000; cat. no. 14695-1-AP), collagen III (1:1,000; cat. no. 22734-1-AP), p-Smad3 (1:1,000; cat. no. 9520), t-Smad3 (1:1,000; cat. no. 9523), p-ERK1/2 (1:1,000; cat. no. 9101), t-ERK1/2 (1:1,000; cat. no. 9102), p-JNK (1:1,000; cat. no. 4671), t-JNK (1:1,000; cat. no. 9252), p-p38 (1:1,000; cat. no. 4511), t-p38 (1:1,000; cat. no. 9212) and GAPDH (1:5,000; cat. no. AG019). Following washing three times with washing buffer (TBS containing $0.1 \%$ Tween-20), the membranes were incubated with horseradish peroxidase (HRP)-conjugated secondary antibodies [HRP-conjugated goat anti-rabbit IgG, 1:2,000, cat. no. D110058; HRP-conjugated goat anti-mouse IgG, 1:2,000, cat. no. D110087; all from Sangon Biotech (Shanghai) Co., Ltd.] for $1 \mathrm{~h}$ at $25^{\circ} \mathrm{C}$. The protein signals were detected using Immobilon ECL HRP Substrate (Merck KGaA), and images were acquired using Chemidoc XRS (Bio-Rad Laboratories, Inc.). Image Pro-Plus 6.0 software (Media Cybernetics, Inc.) was used to quantify the density of the bands.

Immunofluorescence staining. In order to detect the effect of ATV on the trans-differentiation of hVFs into activated myofibroblasts, cells were stained with the fibroblast activation marker $\alpha$-SMA. The fibroblasts $\left(1 \times 10^{4}\right.$ cells/well) were cultured on coverslips in the plate and were subjected to different treatments as follows: i) Control group (DMSO treatment); ii) TGF- $\beta 1$ group; iii) ATV group; and iv) TGF- $\beta 1$ combined with ATV group. Following treatment, cells were washed three times 
Table I. Sequences of primers used for reverse transcription-quantitative PCR.

\begin{tabular}{lll}
\hline Gene & \multicolumn{1}{c}{ Forward sequence $\left(5^{\prime}-3^{\prime}\right)$} & \multicolumn{1}{c}{ Reverse sequence $\left(5^{\prime}-3^{\prime}\right)$} \\
\hline$\alpha-S M A$ & CTATGAGGGCTATGCCTTGCC & GCTCAGCAGTAGTAACGAAGGA \\
MMP-2 & GATACCCCTTTGACGGTAAGGA & CCTTCTCCCAAGGTCCATAGC \\
COL-I & GAGGGCCAAGACGAAGACATC & CAGATCACGTCATCGCACAAC \\
COL-III & GCCAAATATGTGTCTGTGACTCA & GGGCGAGTAGGAGCAGTTG \\
GAPDH & GGAGCGAGATCCCTCCAAAAT & GGCTGTTGTCATACTTCTCATGG
\end{tabular}

$\alpha$-SMA, $\alpha$-smooth muscle actin; MMP-2, matrix metalloproteinase-2; COL-I, type I collagen; COL-III, type III collagen.

with cold PBS, treated with $4 \%$ paraformaldehyde for $20 \mathrm{~min}$ at room temperature and permeabilized with $0.2 \%$ Triton-X 100 for $15 \mathrm{~min}$ at room temperature. Following blocking with 5\% bovine serum albumin [cat. no. A602449; Sangon Biotech (Shanghai) Co., Ltd.] in PBS for $1 \mathrm{~h}$ at room temperature, the slides were stained with the aforementioned primary antibody against $\alpha$-SMA (1:100) overnight at $4^{\circ} \mathrm{C}$. Subsequently, an Alexa Fluor 488-conjugated secondary antibody (1:400; cat. no. A11008; Invitrogen; Thermo Fisher Scientific, Inc.) was used to probe the primary antibody, and the nuclei were stained with DAPI at room temperature for $5 \mathrm{~min}$. Lastly, the cells were covered with antifade mounting medium (cat. no. MM1401; Shanghai Maokang Biotechnology Co., Ltd.), and images were captured using an Olympus fluorescence microscope (Olympus Corporation; magnification, $\mathrm{x} 200$ ).

Reverse transcription-quantitative PCR (RT-qPCR). Total RNA was extracted from cells using NucleoZOL reagent (cat. no. 740404.200; Macherey-Nagel $\mathrm{GmbH}$ ) and was reverse transcribed into cDNA with PrimeScript ${ }^{\mathrm{TM}}$ RT Master mix (cat. no. RR036A; Takara Biotechnology Co., Ltd.) according to the manufacturer's protocol. Firstly, ordinary PCR was performed using Rapid Taq Master mix (cat. no.P222; Vazyme Biotech Co., Ltd), according to the manufacturer's protocol, to ensure that the PCR product of each primer pair was specific. The temperature conditions were $95^{\circ} \mathrm{C}$ for $3 \mathrm{~min}$, followed by 30 cycles of $95^{\circ} \mathrm{C}$ for $15 \mathrm{sec}, 60^{\circ} \mathrm{C}$ for $15 \mathrm{sec}$ and $72^{\circ} \mathrm{C}$ for $15 \mathrm{sec}$, and a final extension at $72^{\circ} \mathrm{C}$ for $5 \mathrm{~min}$. Then, $\mathrm{qPCR}$ was performed on a Bio-Rad CFX Connect Real-Time PCR Detection system (Bio-Rad Laboratories, Inc.) using SYBR Green Supermix (cat. no. RR820A; Takara Biotechnology Co., Ltd.). The thermocycling conditions for $\mathrm{qPCR}$ were as follows: Initial denaturation at $95^{\circ} \mathrm{C}$ for $30 \mathrm{sec}$; followed by 40 cycles of $95^{\circ} \mathrm{C}$ for $5 \mathrm{sec}, 60^{\circ} \mathrm{C}$ for $30 \mathrm{sec}$ and $72^{\circ} \mathrm{C}$ for $1 \mathrm{~min}$. GAPDH was used as a reference gene for normalization. The relative gene expressions were calculated using the $2^{-\Delta \Delta \mathrm{Cq}}$ method (22). The primer sequences are presented in Table I.

Statistical analysis. All experiments were performed independently a minimum of three times. The data are presented as the mean \pm standard error of mean. Statistical analysis was performed using GraphPad Prism 7.0 software (GraphPad Software, Inc.). Statistical differences were assessed using one-way analysis of variance followed by Tukey's post hoc test for multiple comparisons. $\mathrm{P}<0.05$ was considered to indicate a statistically significant difference.
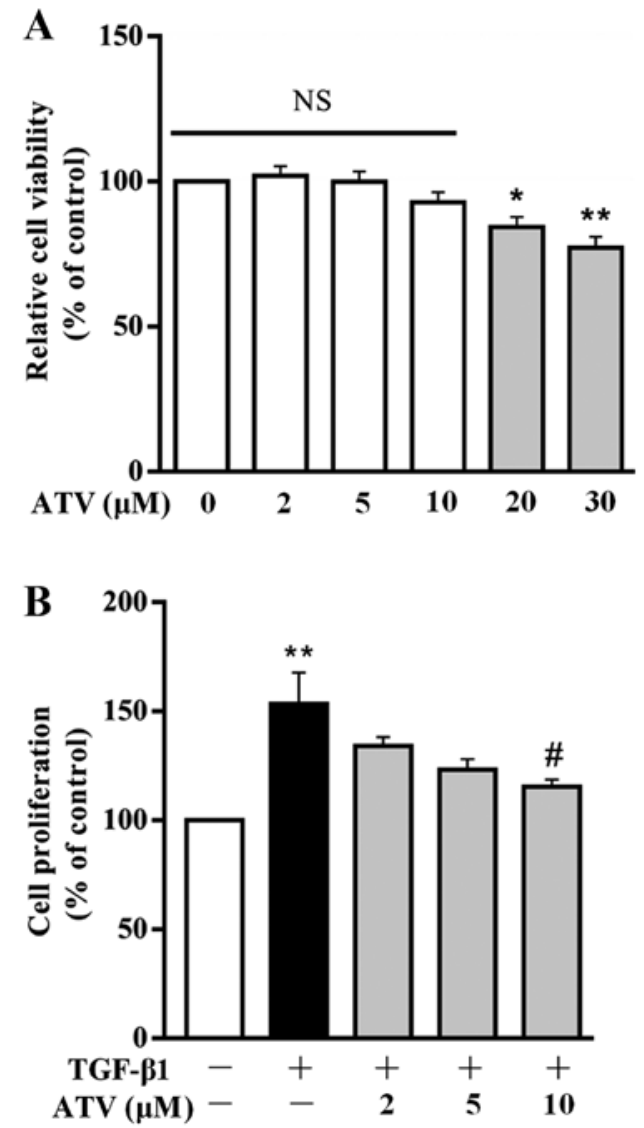

Figure 1. Effect of ATV on TGF- $\beta 1$-induced proliferation of hVFs. (A) Cells were exposed to different concentrations of ATV, and then the viability of the cells was assessed using a CCK-8 assay. (B) hVFs were pretreated with various concentrations of $\operatorname{ATV}(0,2,5$ and $10 \mu \mathrm{M})$ for $3 \mathrm{~h}$, and then exposed to $5 \mathrm{ng} / \mathrm{ml}$ TGF- $\beta 1$ for $24 \mathrm{~h}$. The proliferation of hVFs was then assessed by CCK-8 assay. All data are presented as the mean \pm standard error of mean of three independent experiments and described as a percentage of the control group. ${ }^{*} \mathrm{P}<0.05,{ }^{* *} \mathrm{P}<0.01$ vs. untreated control group. ${ }^{*} \mathrm{P}<0.05$ vs. TGF- $\beta 1$ only-treated group. hVFs, human ventricular fibroblasts; ATV, atorvastatin; TGF- $\beta 1$, transforming growth factor- $\beta 1$; NS, not significant; CCK-8, Cell Counting Kit-8.

\section{Results}

ATV suppresses TGF- $\beta 1$-induced proliferation of $h V F$ s. Firstly, to investigate the cytotoxic effect of ATV on hVFs, cells were incubated with ATV for $24 \mathrm{~h}$ at different concentrations $(0,2$, $5,10,20$ and $30 \mu \mathrm{M})$, and then cell viability was detected using a CCK-8 assay. As presented in Fig. 1A, ATV did not induce 


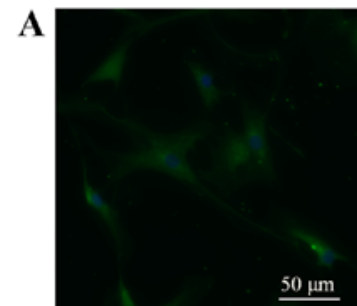

Control

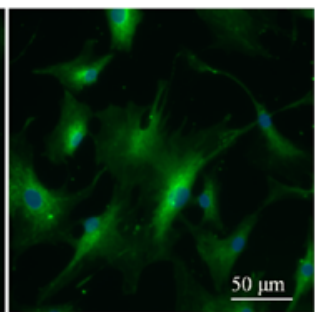

TGF- 11

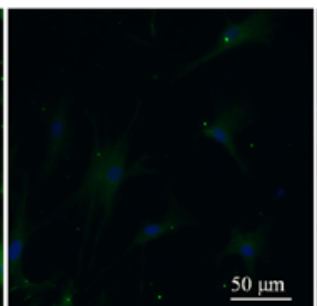

ATV

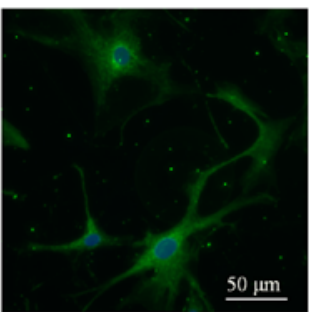

ATV+TGF- $\beta 1$

C

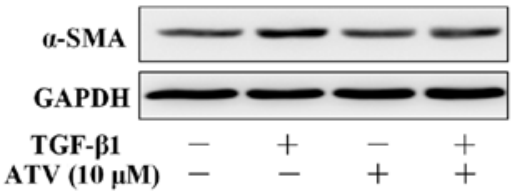

B

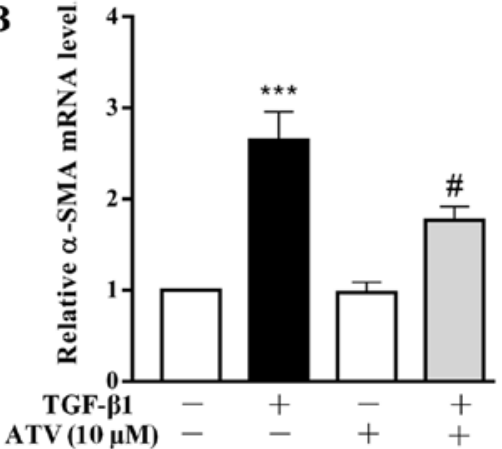

D

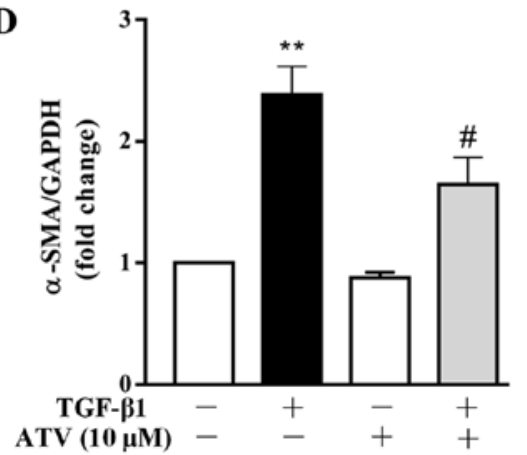

Figure 2. ATV inhibits the trans-differentiation of hVFs into myofibroblasts. Cells were exposed to ATV $(10 \mu \mathrm{M})$ for $3 \mathrm{~h}$ and then co-treated with $5 \mathrm{ng} / \mathrm{ml}$ TGF- $\beta 1$ for a further $24 \mathrm{~h}$. (A) Cells were then immunostained with anti- $\alpha$-SMA antibody (green) and stained with DAPI (nuclei; blue). Magnification, x200. (B) The $\alpha$-SMA mRNA level was assessed by reverse transcription-quantitative PCR assay. (C) The protein expression of $\alpha$-SMA was measured by western blotting. (D) Quantitative analysis of $\alpha$-SMA protein level in hVFs. The relative value was normalized to GAPDH expression. The data are presented as the mean \pm standard error of mean of three independent experiments. ${ }^{* *} \mathrm{P}<0.01,{ }^{* * * *} \mathrm{P}<0.001$ vs. untreated control group. ${ }^{*} \mathrm{P}<0.05$ vs. TGF- $\beta 1$ only-treated group. ATV, atorvastatin; TGF- $\beta 1$, transforming growth factor- $\beta 1$; hVFs, human ventricular fibroblasts; $\alpha$-SMA, $\alpha$-smooth muscle actin.

cytotoxicity at concentrations $\leq 10 \mu \mathrm{M}$. Subsequently, to assess the effect of ATV on the TGF- $\beta 1$-stimulated proliferation of $\mathrm{hVFs}$, cells were exposed to $5 \mathrm{ng} / \mathrm{ml} \mathrm{TGF}-\beta 1$ for $24 \mathrm{~h}$ after pre-incubation with ATV $(0,2,5$ and $10 \mu \mathrm{M})$ for $3 \mathrm{~h}$. The rate of proliferation was then detected using the CCK-8 assay and compared with that of the control group. The results demonstrated that TGF- $\beta 1$ significantly increased the proliferation of hVFs. However, pre-incubation with ATV $(10 \mu \mathrm{M})$ significantly attenuated the increase in cell proliferation induced by TGF- $\beta 1$ (Fig. 1B). Therefore, $10 \mu \mathrm{M}$ was selected as the concentration of ATV for the following experiments.

ATV treatment prevents the trans-differentiation of $h V F s$ into myofibroblasts. Several studies have demonstrated that the differentiation of CFs into myofibroblasts plays a central role in the development of cardiac fibrosis $(23,24)$. The marker of fibroblast activation is the overexpression of $\alpha$-SMA (25). Therefore, the effect of ATV on TGF- $\beta 1$-induced $\alpha$-SMA expression in hVFs was assessed. Firstly, $\alpha$-SMA expression was detected by immunofluorescence staining. As presented in Fig. 2A, in comparison with that of the control group, treatment with TGF- $\beta 1$ alone for $24 \mathrm{~h}$ increased the expression level of $\alpha$-SMA in hVFs. However, pre-incubation with ATV markedly inhibited the TGF- $\beta 1$-induced $\alpha$-SMA expression in hVFs. To confirm the immunostaining results, $\alpha$-SMA expression levels were further detected using RT-qPCR and western blotting. Consistently, the significant upregulation of $\alpha$-SMA in hVFs exposed to TGF- $\beta 1$ was significantly attenuated by pre-incubation with ATV at both the mRNA and protein levels (Fig. 2B-D).

ATV inhibits TGF- $\beta 1$-induced ECM production in $h V F s$. It has been reported that ECM deposition plays an important role in the pathogenesis of myocardial fibrosis (26). Thus, the effects of ATV on TGF- $\beta 1$-stimulated ECM expression in hVFs were further investigated. Both RT-qPCR and immunoblotting assays revealed that TGF- $\beta 1$ significantly increased the expression levels of MMP-2, collagen I and collagen III in hVFs. Whereas, pretreatment of hVFs with ATV significantly attenuated the TGF- $\beta 1$-induced increases in the expression levels of these ECM components (Fig. 3A-C).

ATV inhibits TGF- $\beta 1$-induced activation of the Smad3 and MAPK signaling pathways in hVFs. To investigate the signaling pathways that are involved in the ATV-suppressed fibrotic response in TGF- $\beta 1$-treated $\mathrm{hVF}$, the effect of ATV on the activation of Smad3, ERK1/2, p38 and JNK signaling was detected. As presented in Fig. 4A-D, the western blot results indicated that the phosphorylation of Smad3, ERK1/2, JNK and p38 was significantly increased in TGF- $\beta 1$-induced hVFs. 

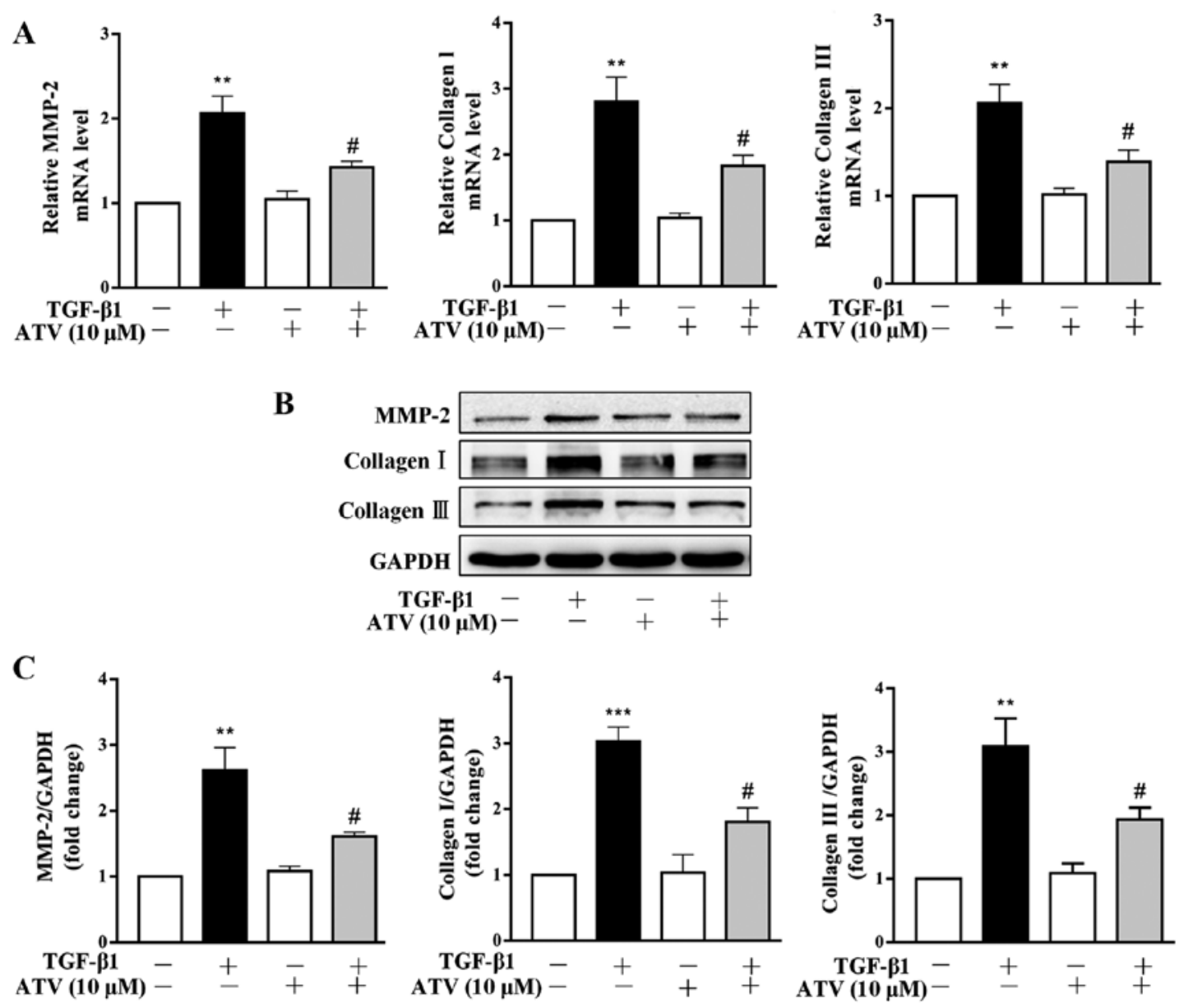

Figure 3. ATV inhibits TGF- $\beta 1$-stimulated ECM production in hVFs. Fibroblasts were pretreated with ATV $(10 \mu \mathrm{M})$ for $3 \mathrm{~h}$ and then co-stimulated with $5 \mathrm{ng} / \mathrm{ml}$ TGF- $\beta 1$ for a further $24 \mathrm{~h}$. (A) The mRNA levels of MMP-2, type I collagen and type III collagen were assessed by reverse transcription-quantitative PCR. (B) The protein expression levels of MMP-2, collagen I and collagen III were detected by western blotting. (C) Quantitative analysis of the western blot results was performed by evaluating the protein band densities with Image Pro-Plus version 6.0 software. The relative value was normalized to GAPDH expression. All data are expressed as the mean \pm standard error of mean of three independent experiments. ${ }^{* *} \mathrm{P}<0.01,{ }^{* * * *} \mathrm{P}<0.001$ vs. untreated control group. ${ }^{\#} \mathrm{P}<0.05$ vs. TGF- $\beta 1$ only-treated group. ATV, atorvastatin; TGF- $\beta 1$, transforming growth factor- $\beta 1$; ECM, extracellular matrix; hVFs, human ventricular fibroblasts; MMP-2, matrix metalloproteinase-2.

However, the TGF- $\beta 1$-induced activation of these signaling molecules was significantly suppressed by ATV. These data demonstrated that the ATV-decreased fibrotic response in hVFs treated by TGF- $\beta 1$ was likely associated with suppression of Smad3 and MAPK signaling (Fig. 5).

\section{Discussion}

To date, several specific anti-fibrotic therapies have been evaluated for use, with unsatisfactory results (27). Therefore, identifying a novel and effective strategy for the treatment of cardiac fibrosis is urgently required. To the best of our knowledge, the present data indicated for the first time that ATV prevents TGF- $\beta 1$-stimulated myofibroblast differentiation and ECM protein production in $\mathrm{hVFs}$, and the underlying mechanism may be, at least in part, due to the suppression of Smad3 and MAPK signaling activation.

CFs account for $2 / 3$ of the total cell number in the adult human heart, and contribute to the maintenance of cardiac structure, function and ECM homeostasis in the normal state, and the regulation of structural integrity and scar formation following myocardial injury $(28,29)$. Abnormal proliferation of
CFs is the major cellular pathological basis of cardiac fibrosis. Increasing evidence suggests that TGF- $\beta 1$, a key pro-fibrotic cytokine, can promote $\mathrm{CF}$ proliferation $(30,31)$, which was confirmed in the present study. Furthermore, TGF- $\beta 1$ has been demonstrated to be markedly and consistently activated in various cardiovascular diseases, including myocardial hypertrophy and myocardial infarction $(32,33)$. The findings of the present study indicated that ATV effectively decreased cell proliferation in TGF- $\beta 1$-stimulated hVFs. The results revealed that ATV can exert protective effects against myocardial fibrosis through suppressing hVF proliferation.

The trans-differentiation of CFs into myofibroblasts has been shown to play a vital role in the progress of cardiac fibrosis (34). Myofibroblasts are defined by excessive $\alpha$-SMA expression and ECM protein secretion $(5,25)$. The present study demonstrated that TGF- $\beta 1$ significantly promoted fibroblast differentiation and ECM protein expression, which is in accordance with the findings reported by previous studies $(30,31,35)$. However, pre-incubation with ATV inhibited the TGF- $\beta 1$-stimulated fibrotic response in hVFs. The results were in agreement with earlier studies, which reported that ATV prevents advanced glycation end 
A

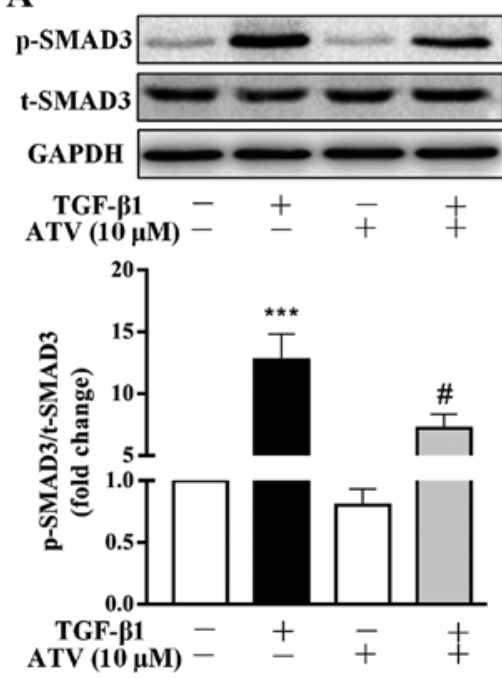

C

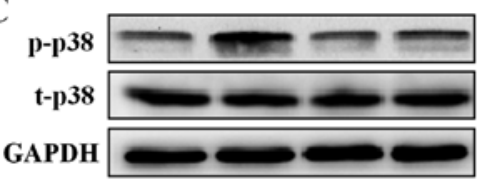

$\underset{\operatorname{TTV}(10 \mu \mathrm{MM})}{\operatorname{TH}}-\stackrel{+}{+}+$

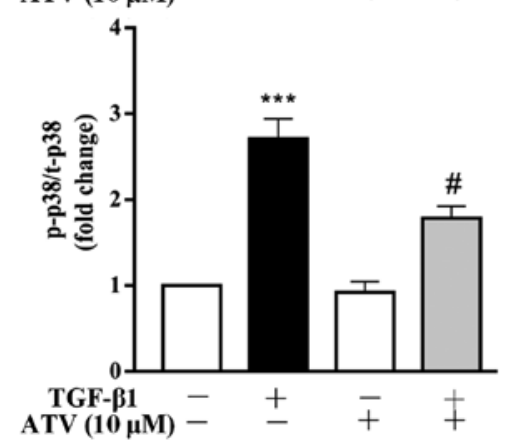

B

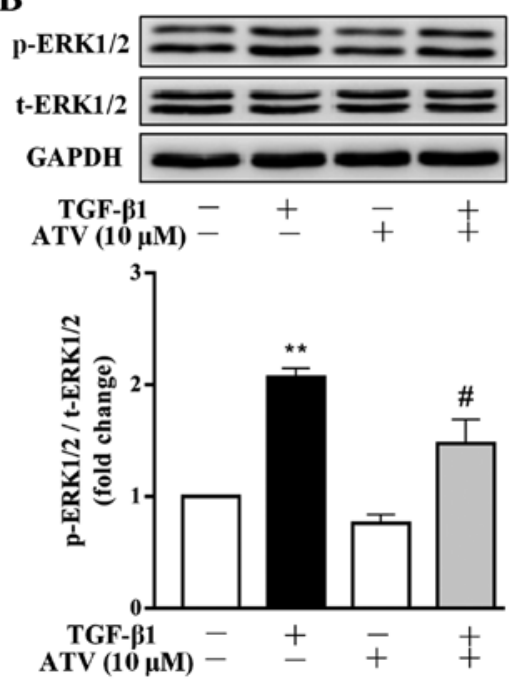

D

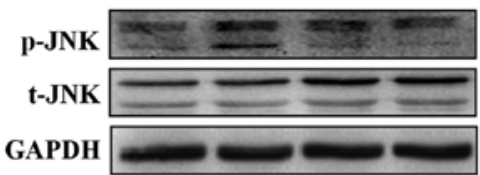

$\underset{\operatorname{TGF}-\beta 1}{\operatorname{TV}(10 \mu \mathrm{M})}-\frac{+}{+} \quad+$

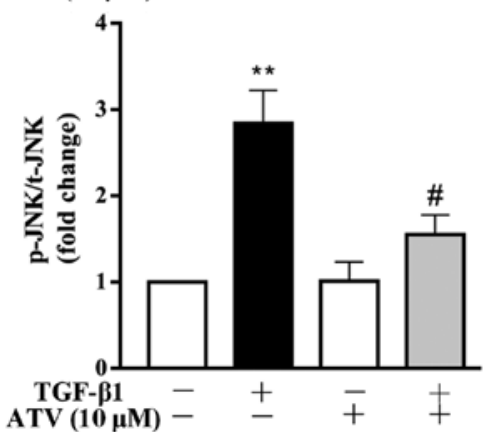

Figure 4. ATV inhibits TGF- $\beta 1$-induced Smad 3 and MAPK signaling activation in hVFs. Fibroblasts were pre-incubated with ATV (10 $\mu \mathrm{M})$ for $3 \mathrm{~h}$ and then co-treated with $5 \mathrm{ng} / \mathrm{ml} \mathrm{TGF- \beta 1} \mathrm{for} 30 \mathrm{~min}$. The protein levels of (A) p-Smad3 and t-Smad3, (B) p-ERK1/2 and t-ERK1/2, (C) p-p38 and t-p38, and (D) p-JNK and $t-J N K$ were detected using western blotting. The relative density was expressed as the ratio of p-protein to the corresponding t-protein. Values represent the mean \pm standard error of mean of three independent experiments. ${ }^{* *} \mathrm{P}<0.01,{ }^{* * *} \mathrm{P}<0.001$ vs. untreated control group. ${ }^{*} \mathrm{P}<0.05$ vs. TGF- $\beta 1$ only-treated group. ATV, atorvastatin; TGF- $\beta 1$, transforming growth factor- $\beta 1$; MAPK, mitogen-activated protein kinase; hVFs, human ventricular fibroblasts; $\mathrm{p}$-, phosphorylated; t-, total; ERK1/2, extracellular signal-regulated kinase 1/2; JNK, c-Jun N-terminal kinase.

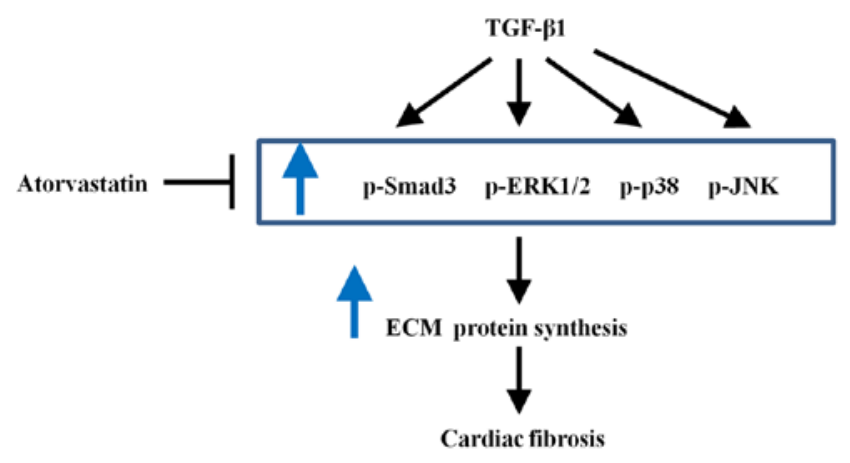

Figure 5. Schematic diagram demonstrating how atorvastatin inhibits TGF- $\beta 1$-induced fibrogenesis in hVFs. TGF- $\beta 1$ treatment can induce activation of the Smad3 and MAPK signaling pathways, cause an increase in ECM synthesis and promote the fibrogenesis in hVFs. Atorvastatin plays a protective role and can prevent TGF- $\beta 1$-induced cardiac fibrosis by inhibiting the activation of Smad3 and MAPK signaling pathways in hVFs. MAPK, mitogen-activated protein kinase; hVFs, human ventricular fibroblasts; ECM, extracellular matrix; p-, phosphorylated; ERK1/2, extracellular signal-regulated kinase 1/2; JNK, c-Jun N-terminal kinase; TGF- $\beta 1$, transforming growth factor- $\beta 1$. products-, angiotensin II-, hypertension-, aldosterone- and doxorubicin-induced cardiac fibrosis (16-20).

TGF- $\beta 1$ canonically activates downstream Smad pathways, and TGF- $\beta 1 /$ Smad signaling plays a crucial role in the development of myocardial fibrosis (36-38). During fibrogenesis, Smad3, a major downstream mediator of TGF- $\beta 1$, is phosphorylated by activated TGF- $\beta$ type I receptor kinase (39-41). $\mathrm{p}$-Smad3 protein then interacts with Smad4 to form a complex and translocates to the cell nucleus, where it functions as a transcriptional factor and induces the transcriptional activation of numerous fibrotic genes, including $\alpha$-SMA, MMP-2 and collagens $(7,8)$. In addition to the Smad-mediated pathways, TGF- $\beta 1$ can also act through non-canonical signaling pathways, such as the MAPK signaling pathway (9). Accumulating evidence has reported that ERK/JNK/p38 MAPK signaling is involved in regulating multiple cellular processes, including cell proliferation, differentiation and survival $(42,43)$. In addition, MAPK signaling pathways have 
also been shown to play an essential role in the progression of cardiac fibrosis. For example, ERK1/2 has been reported to play an important signaling role in driving $\mathrm{CF}$ proliferation (44), and it is required for TGF- $\beta 1$-induced pro-fibrotic phenotypes (3). Molkentin et al (11) reported that transgenic mice with fibroblast-specific activation of p38 MAPK developed interstitial and perivascular fibrosis in the heart. Thus, inhibiting these signaling pathways may imply a promising therapeutic strategy against cardiac fibrosis. The present study evaluated the importance of Smad3 and MAPK signaling in TGF- $\beta 1$-induced cardiac fibrosis in hVFs. The results indicated that TGF- $\beta 1$ significantly activated both Smad3 and MAPK signaling in hVFs. However, ATV pretreatment significantly decreased the TGF- $\beta 1$-induced phosphorylation levels of Smad3, ERK1/2, JNK and p38 MAPK in hVFs. This suggested that ATV inhibited TGF- $\beta 1$-induced fibrogenesis in hVFs at least in part through inhibition of the Smad3 and MAPK signaling pathways.

There are also some limitations in the present study. Firstly, the results were obtained only from a series of in vitro experiments, and were not validated in vivo. Secondly, the exact targets of ATV in cardiac fibrosis remain unclear. Further investigations need to be performed to resolve these limitations.

Taken together, the results of the present study report a protective role of ATV on TGF- $\beta 1$-induced fibrogenesis in hVFs and the potential mechanism involved. The results demonstrated that ATV prevented TGF- $\beta 1$-induced fibrogenesis in hVFs at least in part by inhibiting Smad3 and MAPK signaling activation. These novel findings suggest a potential therapeutic effect of ATV against fibrotic disease in clinical practice.

\section{Acknowledgements}

Not applicable.

\section{Funding}

This study was supported by The Natural Science Foundation of Southwest Medical University and The Foundation of The Affiliated Hospital of Southwest Medical University (grant no. 2017-PT-43).

\section{Availability of data and materials}

All data used or analyzed during the present study are available from the corresponding author on reasonable request.

\section{Authors' contributions}

YD, HX, JW, XW, TL, JF, SZ, QY and JL performed the experiments. ZF and GL conceived and designed the research. YD, HX, GL and ZF analyzed the data and drafted the manuscript. QY and JL reviewed and edited the manuscript. All authors read and approved the final version of the manuscript.

\section{Ethics approval and consent to participate}

Not applicable.

\section{Patient consent for publication}

Not applicable.

\section{Competing interests}

The authors declare that they have no competing interests.

\section{References}

1. Rockey DC, Bell PD and Hill JA: Fibrosis-a common pathway to organ injury and failure. N Engl J Med 373: 96, 2015.

2. Davis J and Molkentin JD: Myofibroblasts: Trust your heart and let fate decide. J Mol Cell Cardiol 70: 9-18, 2014.

3. Schafer S, Viswanathan S, Widjaja AA, Lim WW, Moreno-Moral A, DeLaughter DM, Ng B, Patone G, Chow K, Khin E, et al: IL-11 is a crucial determinant of cardiovascular fibrosis. Nature 552: 110-115, 2017.

4. Akhurst RJ and Hata A: Targeting the TGF $\beta$ signalling pathway in disease. Nat Rev Drug Discov 11: 790-811, 2012.

5. Wynn TA: Cellular and molecular mechanisms of fibrosis. J Pathol 214: 199-210, 2008.

6. Eghbali M, Tomek R, Woods C and Bhambi B: Cardiac fibroblasts are predisposed to convert into myocyte phenotype: Specific effect of transforming growth factor beta. Proc Natl Acad Sci USA 88: 795-799, 1991.

7. Shi Y and Massague J: Mechanisms of TGF-beta signaling from cell membrane to the nucleus. Cell 113: 685-700, 2003.

8. Schiller M, Javelaud D and Mauviel A: TGF-beta-induced SMAD signaling and gene regulation: Consequences for extracellular matrix remodeling and wound healing. I Dermatol Sci 35: 83-92, 2004.

9. Evangelia $P$ and Peter TD: TGF $\beta$ signaling and cardiovascular diseases. Int J Bio Sci 8: 195-213, 2012.

10. Chung CC, Kao YH, Yao CJ, Lin YK and Chen YJ: A comparison of left and right atrial fibroblasts reveals different collagen production activity and stress-induced mitogen-activated protein kinase signalling in rats. Acta Physiol (Oxf) 220: 432-445, 2017.

11. Molkentin JD, Bugg D, Ghearing N, Dorn LE, Kim P, Sargent MA, Gunaje J, Otsu K and Davis J: Fibroblast-specific genetic manipulation of p38 mitogen-activated protein kinase in vivo reveals its central regulatory role in fibrosis. Circulation 136: 549-561, 2017.

12. McFarlane SI, Muniyappa R, Francisco R and Sowers JR: Clinical review 145: Pleiotropic effects of statins: Lipid reduction and beyond. J Clin Endocrinol Metab 87: 1451-1458, 2002.

13. Wang CY, Liu PY and Liao JK: Pleiotropic effects of statin therapy: Molecular mechanisms and clinical results. Trends Mol Med 14: 37-44, 2008.

14. Ludman A, Venugopal V, Yellon DM and Hausenloy DJ: Statins and cardioprotection-more than just lipid lowering? Pharmacol Ther 122: 30-43, 2009.

15. Liao JK: Effects of statins on 3-hydroxy-3-methylglutaryl coenzyme a reductase inhibition beyond low-density lipoprotein cholesterol. Am J Cardiol 96: 24F-33F, 2005.

16. Chen M, Li H, Wang G, Shen X, Zhao S and Su W: Atorvastatin prevents advanced glycation end products (AGEs)-induced cardiac fibrosis via activating peroxisome proliferator-activated receptor gamma (PPAR- $\gamma$ ). Metabolism 65: 441-453, 2016.

17. Choi SY, Park JS, Roh MS, Kim CR, Kim MH and Serebruany V: Inhibition of angiotensin II-induced cardiac fibrosis by atorvastatin in adiponectin knockout mice. Lipids 52: 415-422, 2017.

18. Fang T, Guo B, Xue L and Wang L: Atorvastatin prevents myocardial fibrosis in spontaneous hypertension via interleukin-6 (IL-6)/signal transducer and activator of transcription 3 (STAT3)/endothelin-1 (ET-1) pathway. Med Sci Monit 25: 318-323, 2019.

19. Wang Q, Cui W, Zhang HL, Hu HJ, Zhang YN, Liu DM and Liu J: Atorvastatin suppresses aldosterone-induced neonatal rat cardiac fibroblast proliferation by inhibiting ERK1/2 in the genomic pathway. J Cardiovasc Pharmacol 61: 520-527, 2013.

20. Gao G, Jiang S, Ge L, Zhang S, Zhai C, Chen W and Sui S: Atorvastatin improves doxorubicin-induced cardiac dysfunction by modulating Hsp70, Akt, and MAPK signaling pathways. J Cardiovasc Pharmacol 73: 223-231, 2019. 
21. Xiao H, Ma X, Feng W, Fu Y, Lu Z, Xu M, Sheng Q, Zhu Y and Zhang Y: Metformin attenuates cardiac fibrosis by inhibiting the TGFbeta1-Smad3 signalling pathway. Cardiovasc Res 87: 504-513, 2010

22. Livak KJ and Schmittgen TD: Analysis of relative gene expression data using real-time quantitative PCR and the 2(-Delta Delta C(T)) method. Methods 25: 402-408, 2001.

23. Yi X, Li X, Zhou Y, Ren S, Wan W, Feng G and Jiang X: Hepatocyte growth factor regulates the TGF- $\beta 1$-induced proliferation, differentiation and secretory function of cardiac fibroblasts. Int J Mol Med 34: 381-390, 2014.

24. Van Nieuwenhoven FA and Turner NA: The role of cardiac fibroblasts in the transition from inflammation to fibrosis following myocardial infarction. Vascul Pharmacol 58: 182-188, 2013.

25. Abdalla M, Goc A, Segar L and Somanath PR: Akt1 mediates $\alpha$-smooth muscle actin expression and myofibroblast differentiation via myocardin and serum response factor. J Biol Chem 288 33483-33493, 2013.

26. Lan TH, Huang XQ and Tan HM: Vascular fibrosis in atherosclerosis. Cardiovasc Pathol 22: 401-407, 2013.

27. Park S, Nguyen NB, Pezhouman A and Ardehali R: Cardiac fibrosis: Potential therapeutic targets. Transl Res 209: 121-137, 2019.

28. Jugdutt BI: Remodeling of the myocardium and potential targets in the collagen degradation and synthesis pathways. Curr Drug Targets Cardiovasc Haematol Disord 3: 1-30, 2003.

29. Souders CA, Bowers SL and Baudino TA: Cardiac fibroblast: The renaissance cell. Circ Res 105: 1164-1176, 2009.

30. Li P, Wang D, Lucas J, Oparil S, Xing D, Cao X, Novak L, Renfrow MB and Chen YF: Atrial natriuretic peptide inhibits transforming growth factor beta-induced Smad signaling and myofibroblast transformation in mouse cardiac fibroblasts. Circ Res 102: 185-192, 2008.

31. Rizvi F, Siddiqui R, DeFranco A, Homar P, Emelyanova L, Holmuhamedov E, Ross G, Tajik AJ and Jahangir A: Simvastatin reduces TGF- 31 -induced SMAD2/3-dependent human ventricular fibroblasts differentiation: Role of protein phosphatase activation. Int J Cardiol 270: 228-236, 2018.

32. Bujak M and Frangogiannis NG: The role of TGF-beta signaling in myocardial infarction and cardiac remodeling. Cardiovasc Res 74: 184-195, 2007.

33. Li RK, Li G, Mickle DA, Weisel RD, Merante F, Luss H, Rao V, Christakis GT and Williams WG: Overexpression of transforming growth factor-betal and insulin-like growth factor-I in patients with idiopathic hypertrophic cardiomyopathy. Circulation 96: 874-881, 1997.
34. Travers JG, Kamal FA, Robbins J, Yutzey KE and Blaxall BC: Cardiac fibrosis: The fibroblast awakens. Circ Res 118: 1021-1040, 2016.

35. Petrov VV, Fagard RH and Lijnen PJ: Stimulation of collagen production by transforming growth factor-betal during differentiation of cardiac fibroblasts to myofibroblasts. Hypertension 39: 258-263, 2002

36. Li B, Chen H, Yang X, Wang Y, Qin L and Chu Y: Knockdown of eIF3a ameliorates cardiac fibrosis by inhibiting the TGF- $\beta 1 / \mathrm{Smad} 3$ signaling pathway. Cell Mol Biol (Noisy-le-grand) 62: 97-101, 2016.

37. Zhang M, Pan X, Zou Q, Xia Y, Chen J, Hao Q, Wang H and Sun D: Notch3 Ameliorates cardiac fibrosis after myocardial infarction by inhibiting the TGF- $\beta 1 / \mathrm{Smad} 3$ pathway. Cardiovasc Toxicol 16: 316-324, 2016.

38. Zhao M, Zheng S, Yang J, Wu Y, Ren Y, Kong X, Li W and Xuan J: Suppression of TGF- $\beta 1 /$ Smad signaling pathway by sesamin contributes to the attenuation of myocardial fibrosis in spontaneously hypertensive rats. PLoS One 10: e0121312, 2015.

39. Khalil H, Kanisicak O, Prasad V, Correll RN, Fu X, Schips T, Vagnozzi RJ, Liu R, Huynh T, Lee SJ, et al: Fibroblast-specific TGF- $\beta$-Smad $2 / 3$ signaling underlies cardiac fibrosis. J Clin Invest 127: 3770-3783, 2017.

40. Massagué J: TGF $\beta$ signalling in context. Nat Rev Mol Cell Biol 13: 616-630, 2012.

41. Schmierer B and Hill CS: TGFbeta-SMAD signal transduction: Molecular specificity and functional flexibility. Nat Rev Mol Cell Biol 8: 970-982, 2007.

42. Lee SJ, Park K, Ha SD, Kim WJ and Moon SK: Gleditsia sinensis thorn extract inhibits human colon cancer cells: The role of ERK1/2, G2/M-phase cell cycle arrest and p53 expression. Phytother Res 24: 1870-1876, 2010.

43. Yin Y, Guan Y, Duan J, Wei G, Zhu Y, Quan W, Guo C, Zhou D, Wang Y, Xi M and Wen A: Cardioprotective effect of Danshensu against myocardial ischemia/reperfusion injury and inhibits apoptosis of H9c2 cardiomyocytes via Akt and ERK1/2 phosphorylation. Eur J Pharmacol 699: 219-226, 2013.

44. Zhang W and Liu HT: MAPK signal pathways in the regulation of cell proliferation in mammalian cells. Cell Res 12: 9-18, 2002.

(7) $\ominus$ This work is licensed under a Creative Commons Attribution-NonCommercial-NoDerivatives 4.0 International (CC BY-NC-ND 4.0) License. 